\title{
LANTANA CAMARA L. INVASION AND IMPACT ON HERB LAYER DIVERSITY AND SOIL PROPERTIES IN A DRY DECIDUOUS FOREST OF INDIA
}

\author{
SHARMA, G.P. ${ }^{1,2} *$-RAGHUBANSHI, A.S. ${ }^{1}$ \\ ${ }^{1}$ Department of Botany, Banaras Hindu University, Varanasi 221005, India \\ ${ }^{2}$ Department of Environmental Studies, University of Delhi, Delhi 110007, India \\ *Corresponding author \\ e-mail: gyanprakashsharma@gmail.com \\ (Received $31^{\text {st }}$ March 2008; accepted $8^{\text {th }}$ July 2011)
}

\begin{abstract}
Invasion of lantana (Lantana camara L.) in dry deciduous forest is capable of causing changes in micro sites (soil properties and species composition) in which they invade. As lantana is most conspicuous invader in the Vindhyan dry deciduous forests of India. We analyzed the effect of this invasive species on the composition of herbaceous layer and on soil properties. Habitats with different level of canopy cover were analyzed. And the canopy cover was significantly related to the lantana cover. Results indicate that different levels of lantana cover affect soil properties and herbaceous species composition. We found as the lantana cover increases some of the species get locally extinct and some are favored by lantana invasion. Principal Component Analysis (PCA) ordination indicated that the quadrates with nil and differently covered lantana were unique with herb species composition, especially the sites with no lantana cover had native species which were not present in site with differently covered lantana. The distinctness of herb species composition is indicative of marked spatial dynamics with regard to nil and differently covered lantana. Concentration of organic carbon (OC) and total nitrogen (TN) were significantly higher in habitats having large lantana cover. Thus we may conclude that lantana modifies the spatial pattern of herbaceous plant species and the nutrient levels of soil.
\end{abstract}

Keywords: Lantana camara, local extinction, soil properties, species composition

\section{Introduction}

Exotic species invasion is amongst the most important global scale problems experienced by natural ecosystems and is also considered as the second largest threat to global biodiversity (Drake et al., 1989). In India, dry tropical accounts for 38.2\% of the total forest cover (MoEF, 1999). These forests are under immense anthropogenic pressure in form of rapid industrialization and related land-use change in the past few decades. With increase in human population forests are also exposed to illegal sporadic tree felling, widespread lopping of trees for timber resources and shrubs for fuel wood or leaf fodder (Singh and Singh 1989; Jha and Singh, 1990). All these have lead to forest fragmentation, which is prone to subsequent invasion by exotic species (Tripathi, 2003). Invasion of species may lead to local declines (Islam, 2001) and even extinction of native species (Pimm, 1986) thus altering species richness in the forest fragment (Carey et al., 1996). We studied Lantana (Lantana camara L.) as it has spread in almost all the fragmented areas in the Vindhyan dry deciduous forest, and has been ranked as the highest impacting invasive species (Batianoff and Butler, 2003), and is among the 100 worlds worst invasive alien species (GISP, 2003), because it posses great potential to escape cultivation and have deleterious effect on species richness (Islam, 2001). In India it was introduced in early nineteenth century as an ornamental plant (Sharma, 1988), but now it is growing densely throughout India (Sharma et al., 2005a,b). 
However, small-scale environmental changes caused by lantana invasion have not been addressed in dry tropical forest areas. The objective of the present study was to analyze the effect of lantana invasion on the composition of the herbaceous layer and on the soil properties of the dry deciduous forest.

\section{Materials and methods}

\section{Study area}

The study area (Fig 1) lies on the Vindhyan plateau in the Sonebhadra district of Uttar Pradesh $\left(24^{\circ} 6^{\prime}\right.$ to $24^{\circ} 21^{\prime} \mathrm{N} ; 82^{\circ} 59^{\prime}$ to $\left.83^{\circ} 14^{\prime}\right)$. The elevation above the mean sea level ranges between 315 and $485 \mathrm{~m}$ (Singh and Singh, 1992).

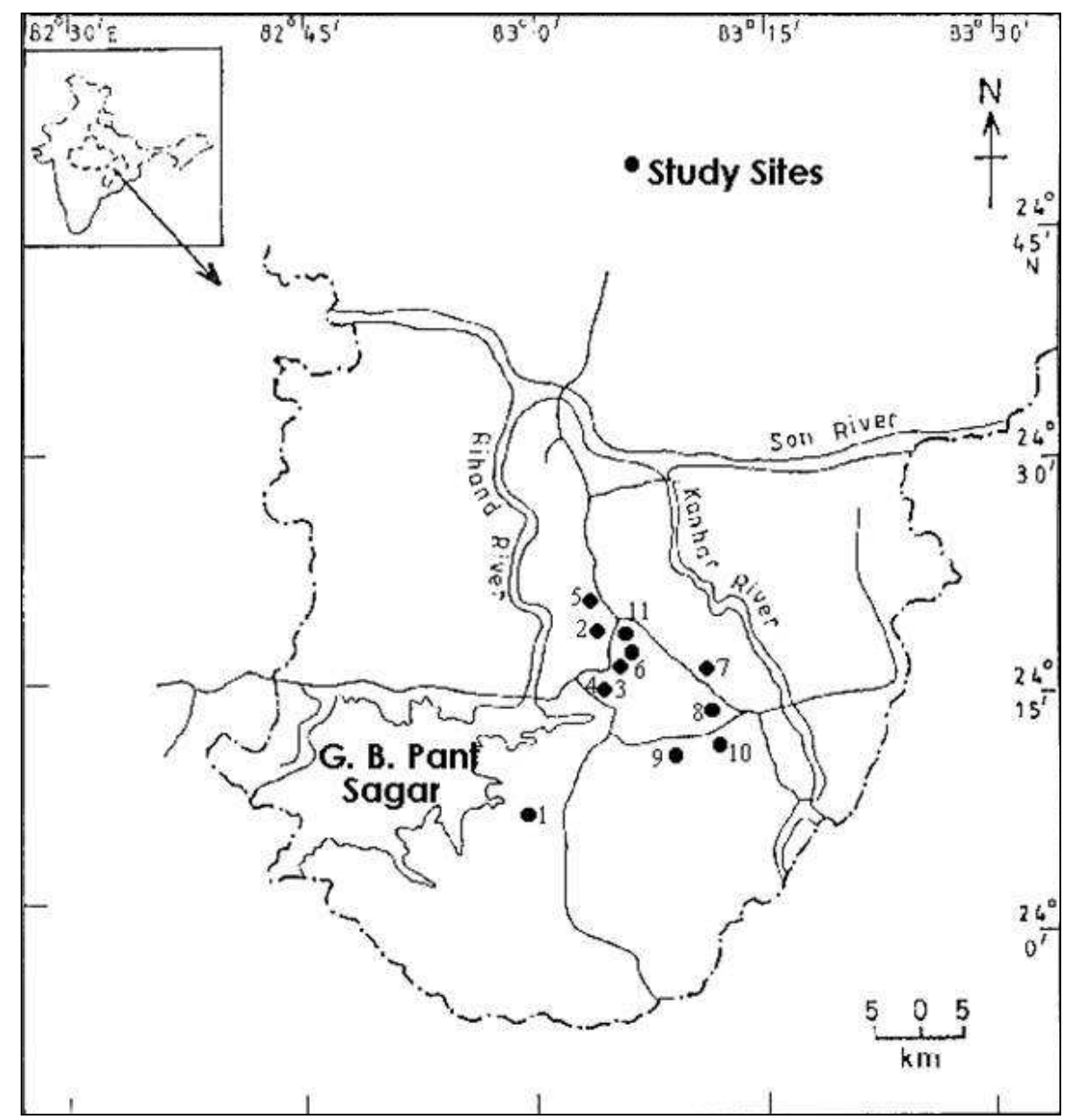

Figure 1. Location of study sites within Vindhyan highlands, India.

1- Khatabaran; 2- Hathwani I; 3- Hathwani II; 4- Kadhpathar; 5- Majhauli;

6-Hathinala I;7- Bhaheradol; 8-Rajkhar; 9-Manbasa; 10-Runtola; 11- Hathinala II

The climate is tropical with three seasons in a year, i.e. summer (from March to mid June), rainy (mid June to September) and winter (October to February). October and March constitute the transition months between the rainy and winter seasons, and between winter and summer seasons, respectively. The average rainfall varies between 850 and $1300 \mathrm{~mm}$. About $85 \%$ of the annual rainfall occurs during the rainy season from the southwest monsoon. The maximum monthly temperature varies between $20^{\circ} \mathrm{C}$ 
in January to $46^{\circ} \mathrm{C}$ in June, and the mean minimum monthly temperature between $12^{\circ} \mathrm{C}$ in January to $31^{\circ} \mathrm{C}$ in May.

Red coloured and fine textured sandstone (Dhandraul orthoquartzite) is the most important rock of the area. Sandstone is generally underlain by shale and limestone. The soils derived from these rocks are residual ultisoils and are sandy-loam in texture (Raghubanshi, 1992). These soils are part of the hyperthermic formation of typical plinthustults with ustorthents according to VII approximation of the USDA soil nomenclature (Singh et al., 2002).

The potential natural vegetation of the region is tropical dry deciduous forest, which is locally dominated by species such as Acacia catechu, Anogeissus latifolia, Boswellia serrata, Buchanania lanzan, Diospyros melanoxylon, Hardwickia binata, Lagerstroemia parviflora, Madhuca longifolia and Terminalia tomentosa. Height of the locally dominant canopy tree species varies from $6 \mathrm{~m}$ to $14 \mathrm{~m}$ (Troup, 1921) across the sites and the height of the herb layer varies from $2 \mathrm{~cm}$ to $75 \mathrm{~cm}$.

Lantana is a perennial woody shrub and in its naturalized range, lantana forms dense mono-specific thickets 1-4 $\mathrm{m}$ high and $\approx 1-4 \mathrm{~m}$ in diameter (Sharma et al., 2005b), although some of the clumps smother near by trees and reach up-to the height of 8-15 m (Sharma et al., 2005b). Eleven sites were selected at random in the region, within a radius of approximately $10 \mathrm{~km}$. These sites had visually different levels of invasion of lantana, with one as control site i.e. having no lantana. In Vindhyans micro-distribution pattern of lantana occurs in patches as homogenous clumps.

\section{Data collection}

A total of 11 sites were sampled and at each site, three large quadrats (10m x10m) were sampled randomly for vegetation and soil features in the year 2003, i.e. a total of 33 quadrats were sampled in the entire study area. Tree canopy cover in each quadrat was measured by estimating the area, shaded directly by the overhead tree canopy. The values were then transformed into percentage area. All the measurements were taken during mid-day when the sun was approximately perpendicular to the surface.

Lantana cover was estimated using the Domin Krajina scale and was transformed into percentage cover for final analysis (Mueller-Dumbois and Ellenberg, 1974).

The light intensity was measured by LCA-2 battery portable infrared carbon dioxide analyser having PAR (Photosynthetic Active Radiation) sensors (filtered selenium photocell) (ADC Scinokem International, U.K.); the 70-100\% sunlight (measured as PAR at 11.00 am on a cloud-free day) corresponded to $1,600-1,720 \mu \mathrm{mol} \mathrm{m} \mathrm{m}^{-2} \mathrm{~s}^{-1}$. Light intensity was measured above and below the lantana bushes of the total light received (values reported are in percent). Percentage light attenuation below lantana was calculated using formula:

$$
\% \text { light attenuation }=\frac{\text { Light above lantana }- \text { Light beneath lantana }}{\text { Light above lantana }} \times 100
$$

Within each large quadrate, four $(0.5 \times 0.5 \mathrm{~m})$ mini plots were randomly sampled and density, frequency and cover of herbaceous species were estimated. For density individual plants were counted while frequency of species in each large quadrate was calculated using the ratio of total number of mini plots in which the species occurred to the total number of mini plots studied (i.e. 4) in each quadrate and cover was estimated 
using the ground percentage cover of Domin Krajina scale and transformed into percentage cover in the final analysis (Mueller-Dumbois and Ellenberg, 1974).

Importance values were calculated as the sum of the mean values of relative cover, relative density and relative frequency (Phillips, 1959). Soil samples from the upper10 $\mathrm{cm}$ soil layer were collected randomly from three places in each quadrat. Each sample was mixed thoroughly, air dried and sieved through a $2 \mathrm{~mm}$ mesh screen. Fine roots were hand picked. Soil $\mathrm{pH}$ (1:2.5; Soil:Water) was determined by digital $\mathrm{pH}$ meter (model 7025M Titrino, Metrohm ion analysis, Metrohm Ltd. Switzerland), organic carbon was measured by Walkey and Black rapid titration method (Jackson, 1958) and total nitrogen by Gerhardt Kjeldal analyzer (Gerhardt Gmbh, Germany). Soil moisture was measured in the field condition using the Theta probe (Delta-T devices Ltd., England). Sampling was carried out during October, when luxuriant mature herbaceous vegetation is present in the area.

\section{Data analysis}

Diversity was measured by Shannon-Weiner index $\left(\mathrm{H}^{\prime}\right)$ :

$$
\mathrm{H}^{\prime}=-\sum \mathrm{pi} * \ln \mathrm{pi}
$$

where pi is the importance value contributed by the $\mathrm{i}^{\text {th }}$ species.

Relationship between tree canopy cover, lantana cover and soil parameters was analyzed by using SPSS Version 10.0 (SPSS, 1997) software and Shannon-Weiner diversity and Principle Component Analysis was calculated using Biodiversity Pro Version 2.0 (McAleece, 1997). Quadrats were segregated into nil, low, medium and high lantana cover, on the basis of PCA ordination.

\section{Results}

Lantana cover varied between sites and with tree canopy cover (Table 1). For example, when tree canopy cover was $>30 \%$ (Hathwani I, Hathwani II, Hathinala, Baheradol, Rajkhar, Runtola sites), then the lantana cover varied from 27 to $60 \%$ and when the tree canopy cover was $<30 \%$ (Khatabaran, Kadhpather, Majhauli, Manbasa sites) then there was a considerable increase in lantana cover and it varied from 62 to $84 \%$. Lantana cover was negatively related to tree canopy cover (Table 2). At Hathinala II site where the canopy cover was $63 \%$ no lantana was reported.

Mean vales of light intensity above the lantana bushes was $20 \pm 1.73,25.4 \pm 1.94,39$ \pm 2.17 and $63 \pm 3.08$ for nil, low, medium and high lantana cover respectively, on the other, light intensity recorded beneath the lantana bush was $15.4 \pm 1.50,18.3 \pm 2.69$ and $8.0 \pm 2.73$ for low, medium and high lantana cover respectively, of the total light received. The percentage attenuation of light beneath the lantana increased with increasing lantana cover i.e. 40, 53 and $87 \%$ for low, medium and high lantana cover respectively.

Although the lantana cover varied with changes in soil moisture and $\mathrm{pH}$ (Table 1), it was not significantly related to these soil parameters. There was a strong positive linear relation of lantana cover with soil carbon (Table 2). At the maximum (Manbasa site) and minimum (Hathinala II site) lantana cover, organic carbon varied from 3.38 to $1.46 \%$ (Table 1). The lantana cover also had a strong positive linear relation with soil nitrogen (Table 2) which varied from 0.29 to $0.11 \%$ among the sites (Table 1). 
Table 1. Vegetation and soil characteristics at different sites (Values in parenthesis are \pm $S E$ ). Sites are arranged in order of increasing tree canopy cover.

\begin{tabular}{|c|c|c|c|c|c|c|c|c|c|}
\hline Sites & $\begin{array}{c}\text { Tree } \\
\text { canopy } \\
\text { cover } \\
(\%)\end{array}$ & $\begin{array}{c}\text { Lantana } \\
\text { cover } \\
(\%)\end{array}$ & $\begin{array}{c}\text { Total } \\
\text { herb } \\
\text { cover }(\%)\end{array}$ & $\begin{array}{c}\text { Shannon } \\
\text { diversity } \\
\left(H^{\prime}\right)\end{array}$ & \begin{tabular}{|c} 
Soil \\
moisture \\
$(\%)$
\end{tabular} & pH & $\begin{array}{c}\mathrm{C} \\
(\%)\end{array}$ & $\begin{array}{c}\mathbf{N} \\
(\%)\end{array}$ & 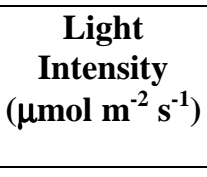 \\
\hline Khatabaran & $\begin{array}{l}15.00 \\
(5.00)\end{array}$ & $\begin{array}{c}75.00 \\
(10.41)\end{array}$ & $\begin{array}{c}33.33 \\
(10.26)\end{array}$ & $\begin{array}{c}0.24 \\
(0.04)\end{array}$ & $\begin{array}{l}12.50 \\
(1.59)\end{array}$ & $\begin{array}{c}7.32 \\
(0.07)\end{array}$ & $\begin{array}{c}2.18 \\
(0.26)\end{array}$ & $\begin{array}{c}0.14 \\
(0.02)\end{array}$ & $\begin{array}{c}1706.6 \\
(8.8)\end{array}$ \\
\hline Manbasa & $\begin{array}{l}20.67 \\
(9.68)\end{array}$ & $\begin{array}{c}83.33 \\
(14.24)\end{array}$ & $\begin{array}{l}32.33 \\
(8.76)\end{array}$ & $\begin{array}{c}0.55 \\
(0.19)\end{array}$ & $\begin{array}{l}14.50 \\
(2.01)\end{array}$ & $\begin{array}{c}7.48 \\
(0.07)\end{array}$ & $\begin{array}{c}3.38 \\
(0.40)\end{array}$ & $\begin{array}{c}0.29 \\
(0.04)\end{array}$ & $\begin{array}{c}1688.3 \\
(6.0)\end{array}$ \\
\hline Majhauli & $\begin{array}{l}26.67 \\
(7.26)\end{array}$ & $\begin{array}{c}68.33 \\
(21.86)\end{array}$ & $\begin{array}{l}17.66 \\
(9.27)\end{array}$ & $\begin{array}{c}0.52 \\
(0.18)\end{array}$ & $\begin{array}{l}20.33 \\
(0.43)\end{array}$ & $\begin{array}{c}7.42 \\
(0.08)\end{array}$ & $\begin{array}{c}2.42 \\
(0.49)\end{array}$ & $\begin{array}{c}0.18 \\
(0.04)\end{array}$ & $\begin{array}{c}1656.6 \\
(14.5)\end{array}$ \\
\hline Kadhpathar & $\begin{array}{c}28.33 \\
(10.93)\end{array}$ & $\begin{array}{c}61.67 \\
(18.56)\end{array}$ & $\begin{array}{c}51.66 \\
(17.81)\end{array}$ & $\begin{array}{c}0.65 \\
(0.13)\end{array}$ & $\begin{array}{l}19.43 \\
(1.22)\end{array}$ & $\begin{array}{c}7.44 \\
(0.08)\end{array}$ & $\begin{array}{c}3.26 \\
(0.44)\end{array}$ & $\begin{array}{c}0.26 \\
(0.05)\end{array}$ & $\begin{array}{c}1616.7 \\
(4.4)\end{array}$ \\
\hline Bhaheradol & $\begin{array}{c}33.33 \\
(18.33)\end{array}$ & $\begin{array}{c}60 \\
(21.79)\end{array}$ & $\begin{array}{c}31.66 \\
(11.09)\end{array}$ & $\begin{array}{c}0.98 \\
(0.48)\end{array}$ & $\begin{array}{l}17.23 \\
(1.37)\end{array}$ & $\begin{array}{c}7.24 \\
(0.08)\end{array}$ & $\begin{array}{c}2.48 \\
(0.64)\end{array}$ & $\begin{array}{c}0.20 \\
(0.06)\end{array}$ & $\begin{array}{l}1536.6 \\
(32.4)\end{array}$ \\
\hline Hathwani I & $\begin{array}{c}36.67 \\
(11.67)\end{array}$ & $\begin{array}{c}48.33 \\
(19.22)\end{array}$ & $\begin{array}{c}70.66 \\
(16.80)\end{array}$ & $\begin{array}{c}0.73 \\
(0.07)\end{array}$ & $\begin{array}{l}15.47 \\
(1.39)\end{array}$ & $\begin{array}{c}7.26 \\
(0.07)\end{array}$ & $\begin{array}{c}1.78 \\
(0.23)\end{array}$ & $\begin{array}{c}0.13 \\
(0.01)\end{array}$ & $\begin{array}{c}1375.0 \\
(22.5)\end{array}$ \\
\hline Runtola & $\begin{array}{c}36.67 \\
(16.91)\end{array}$ & $\begin{array}{c}58.33 \\
(21.67)\end{array}$ & $\begin{array}{l}47.00 \\
(23.50)\end{array}$ & $\begin{array}{c}0.45 \\
(0.25)\end{array}$ & $\begin{array}{l}13.43 \\
(0.88)\end{array}$ & $\begin{array}{c}7.46 \\
(0.05)\end{array}$ & $\begin{array}{c}2.26 \\
(0.35)\end{array}$ & $\begin{array}{c}0.15 \\
(0.02)\end{array}$ & $\begin{array}{l}1295.0 \\
(35.1)\end{array}$ \\
\hline Rajkhar & $\begin{array}{c}45.00 \\
(16.07)\end{array}$ & $\begin{array}{c}56.67 \\
(20.28)\end{array}$ & $\begin{array}{c}36.66 \\
(11.66)\end{array}$ & $\begin{array}{c}0.66 \\
(0.04)\end{array}$ & $\begin{array}{l}18.30 \\
(2.89)\end{array}$ & $\begin{array}{c}7.13 \\
(0.02)\end{array}$ & $\begin{array}{c}2.36 \\
(0.47)\end{array}$ & $\begin{array}{c}0.17 \\
(0.03)\end{array}$ & $\begin{array}{l}956.7 \\
(15.8)\end{array}$ \\
\hline Hathwani II & $\begin{array}{c}51.67 \\
(12.02)\end{array}$ & $\begin{array}{c}56.67 \\
(17.40)\end{array}$ & $\begin{array}{l}21.33 \\
(5.04)\end{array}$ & $\begin{array}{c}0.55 \\
(0.09)\end{array}$ & $\begin{array}{l}17.60 \\
(0.85)\end{array}$ & $\begin{array}{c}7.29 \\
(0.07)\end{array}$ & $\begin{array}{c}2.64 \\
(0.30)\end{array}$ & $\begin{array}{c}0.19 \\
(0.03)\end{array}$ & $\begin{array}{l}859.3 \\
(11.0)\end{array}$ \\
\hline Hathinala I & $\begin{array}{l}56.67 \\
(8.33)\end{array}$ & $\begin{array}{c}26.67 \\
(10.14)\end{array}$ & $\begin{array}{c}73.66 \\
(21.34)\end{array}$ & $\begin{array}{c}0.86 \\
(0.02)\end{array}$ & $\begin{array}{l}18.53 \\
(1.06)\end{array}$ & $\begin{array}{c}7.33 \\
(0.06)\end{array}$ & $\begin{array}{c}1.66 \\
(0.30)\end{array}$ & $\begin{array}{c}0.13 \\
(0.01)\end{array}$ & $\begin{array}{c}784.3 \\
(4.7)\end{array}$ \\
\hline Hathinala II & $\begin{array}{l}63.33 \\
(1.66)\end{array}$ & $\begin{array}{c}0.00 \\
(0.00)\end{array}$ & $\begin{array}{l}94.66 \\
(0.88)\end{array}$ & $\begin{array}{c}1.16 \\
(0.01)\end{array}$ & $\begin{array}{l}18.70 \\
(1.22)\end{array}$ & $\begin{array}{c}7.35 \\
(0.11)\end{array}$ & $\begin{array}{c}1.46 \\
(0.20)\end{array}$ & $\begin{array}{c}0.11 \\
(0.008)\end{array}$ & $\begin{array}{l}707.6 \\
(17.1)\end{array}$ \\
\hline
\end{tabular}

Table 2. Correlation matrix of vegetation and soil parameters in a lantana invaded forest.

\begin{tabular}{|c|c|c|c|c|c|c|c|c|}
\hline & $\begin{array}{c}\text { Canopy } \\
\text { cover } \\
(\%) \\
\end{array}$ & $\begin{array}{c}\text { Herb } \\
\text { layer } \\
\text { diversity }\end{array}$ & $\begin{array}{l}\begin{array}{c}\text { Herb } \\
\text { cover } \\
(\%)\end{array} \\
\end{array}$ & $\begin{array}{c}\text { Lantana } \\
\text { cover } \\
(\%) \\
\end{array}$ & $\begin{array}{c}\text { Carbon } \\
(\%)\end{array}$ & $\begin{array}{c}\text { Nitrogen } \\
(\%)\end{array}$ & pH & $\begin{array}{c}\text { Moisture } \\
(\%)\end{array}$ \\
\hline $\begin{array}{c}\text { Canopy cover } \\
(\%)\end{array}$ & 1 & & & & & & & \\
\hline $\begin{array}{l}\text { Herb layer } \\
\text { diversity }\end{array}$ & $0.662 * *$ & 1 & & & & & & \\
\hline $\begin{array}{l}\text { Herb cover } \\
\quad(\%)\end{array}$ & $0.503 * *$ & $0.612 * *$ & 1 & & & & & \\
\hline $\begin{array}{c}\text { Lantana cover } \\
(\%)\end{array}$ & $-0.853 * *$ & $-0.749 * *$ & $-0.544 * *$ & 1 & & & & \\
\hline Carbon (\%) & $-0.688^{* *}$ & $-0.514 * *$ & -0.256 & $0.824 * *$ & 1 & & & \\
\hline Nitrogen $(\%)$ & $-0.627 * *$ & $-0.409 *$ & -0.202 & $0.743 * *$ & $0.966 * *$ & 1 & & \\
\hline $\mathbf{p H}$ & -0.199 & -0.143 & 0.129 & 0.100 & 0.192 & 0.245 & 1 & \\
\hline Moisture (\%) & 0.202 & 0.276 & 0.136 & -0.159 & 0.060 & 0.052 & -0.094 & 1 \\
\hline
\end{tabular}

\footnotetext{
** significant at level $0.01, *$ significant at level 0.05 (Values are Pearson's correlation coefficients)
} 
The analysis of variance (ANOVA) revealed that the sites differed significantly in terms of canopy, lantana cover, carbon, nitrogen, diversity and herb cover (Table 3).

Maximum herb layer diversity occurred at the Hathinala II site which had no lantana cover followed by Hathinala I site which had $27 \%$ lantana cover, and the minimum occurred at the Khatabaran site where lantana cover was $75 \%$ (Table 1). Lantana cover showed a strong linear negative relationship with Shannon-Weiner diversity index of herbaceous layer (Table 2) and herbaceous cover (Table 2).

Table 3. Summary of ANOVA of different sites for vegetation and soil parameters

\begin{tabular}{|c|c|c|c|c|c|}
\hline Vegetation & & df & Mean Square & $\mathbf{F}$ & $\mathbf{P}$ \\
\hline Canopy cover $(\%)$ & $\begin{array}{l}\text { Between groups } \\
\text { Within groups } \\
\text { Total }\end{array}$ & $\begin{array}{c}3 \\
29 \\
32\end{array}$ & $\begin{array}{c}3649.7 \\
162.2\end{array}$ & 22.50 & 0.000 \\
\hline Herb layer Diversity & $\begin{array}{l}\text { Between groups } \\
\text { Within groups } \\
\text { Total }\end{array}$ & $\begin{array}{c}3 \\
29 \\
32\end{array}$ & $\begin{array}{l}0.536 \\
0.036\end{array}$ & 14.54 & 0.000 \\
\hline Herb cover $(\%)$ & $\begin{array}{l}\text { Between groups } \\
\text { Within groups } \\
\text { Total }\end{array}$ & $\begin{array}{c}3 \\
29 \\
32\end{array}$ & $\begin{array}{c}4000.5 \\
636.0\end{array}$ & 6.29 & 0.002 \\
\hline Lantana Cover (\%) & $\begin{array}{l}\text { Between groups } \\
\text { Within groups } \\
\text { Total }\end{array}$ & $\begin{array}{c}3 \\
29 \\
32\end{array}$ & $\begin{array}{c}11195.23 \\
52.27\end{array}$ & 214.2 & 0.000 \\
\hline Light intensity & $\begin{array}{l}\text { Between groups } \\
\text { Within groups } \\
\text { Total }\end{array}$ & $\begin{array}{c}3 \\
29 \\
32\end{array}$ & $\begin{array}{c}1526668.6 \\
4742.4\end{array}$ & 321.9 & 0.000 \\
\hline \multicolumn{6}{|l|}{ Soil parameters } \\
\hline Carbon $(\%)$ & $\begin{array}{l}\text { Between groups } \\
\text { Within groups } \\
\text { Total }\end{array}$ & $\begin{array}{c}3 \\
29 \\
32\end{array}$ & $\begin{array}{l}4.288 \\
0.240\end{array}$ & 17.88 & 0.000 \\
\hline Nitrogen $(\%)$ & $\begin{array}{l}\text { Between groups } \\
\text { Within groups } \\
\text { Total }\end{array}$ & $\begin{array}{c}3 \\
29 \\
32\end{array}$ & $\begin{array}{l}0.027 \\
0.002\end{array}$ & 11.92 & 0.000 \\
\hline
\end{tabular}

The Principal Component Analysis (PCA) ordination of 33 quadrates on the basis of IVI of herb species is present in Fig 2. The PCA 1 and PCA 2 axis accounted for 14 and $12 \%$ variation respectively. PCA 1 represented the lantana cover $\left(r^{2}=0.36, p=0.05\right)$ cover and the PCA 2 axis was related to tree canopy $\left(\mathrm{r}^{2}=-0.45, \mathrm{p}=0.009\right)$. Fig 2 indicated that the quadrates segregated on the basis of different lantana cover. Revealing that the nil, low, medium and high lantana cover quadrates are behaving differently in terms of herb species composition.

The dominance spectrum of the herbaceous species changed with the increasing level of lantana cover. Alysicarpus vaginalis dominated the herbaceous vegetation at lantana nil sites on the other, Evolvulus alsinoides L. dominated the herbaceous vegetation at low, Oplismenus compositus Beaub. at medium and Corchorus trilocularis L. at high lantana cover (Table 4). Species that occurred at high lantana cover with proportionally greater IVI could be considered lantana tolerant species (Cassia tora L., Corchorus trilocularis L, Echinochloa colona Link., Ichnocarpus frutescens (L.) R. Br., Ludwigia perennis L.) and species that were more predominant at low lantana cover may be considered lantana intolerant (Dichanthium annulatum Stapf., Evolvulus alsinoides L., 
Leucas aspera Spr., Sida acuta Burm. F., S. cordifolia L., S. rhombifolia L., Tephrosia purpurea Pers.).

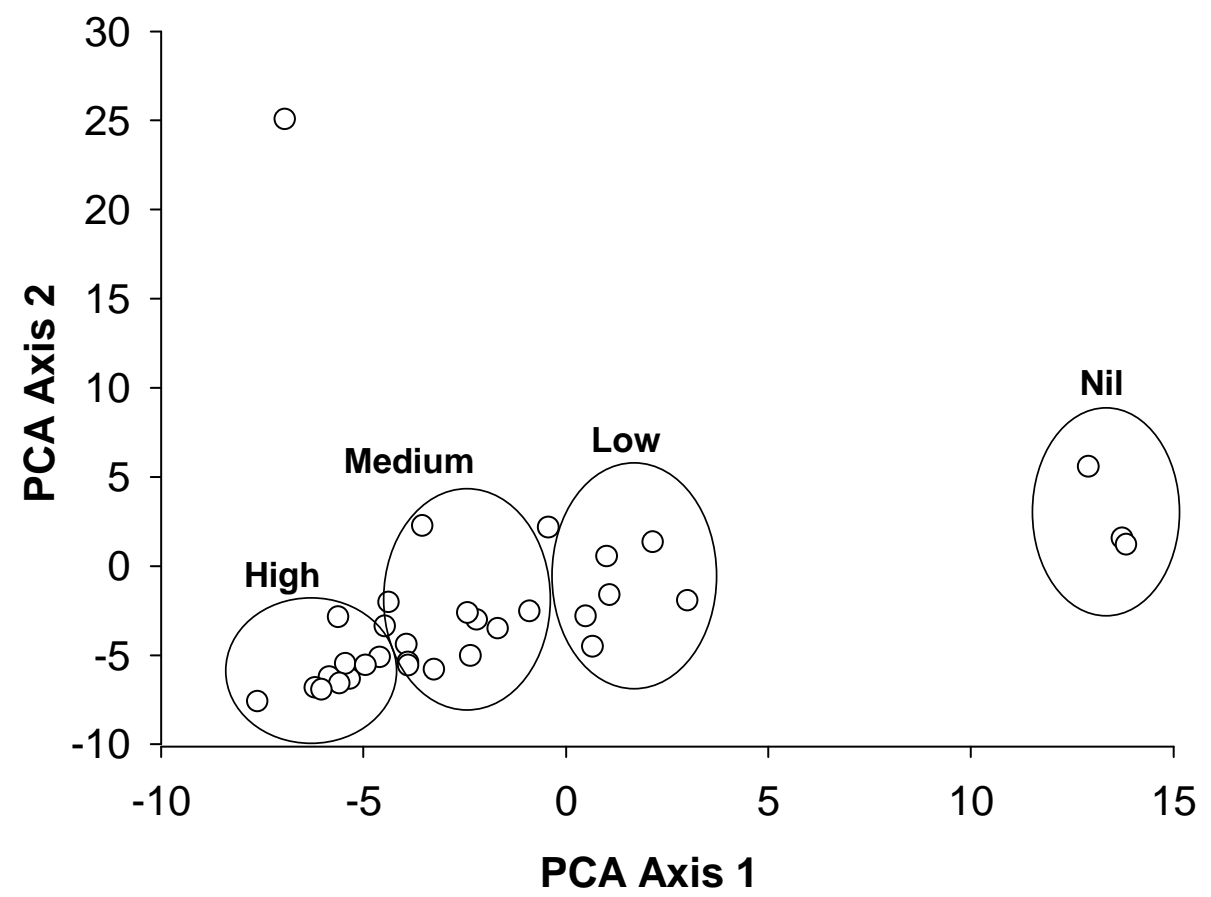

Figure 2. PCA ordination of herbaceous species showing segregation of quadrats at different level of lantana cover (nil, low, medium and high).

The PCA 1 and PCA 2 axis accounted for 14 and $12 \%$ variation respectively.

Species which are unique to the no lantana site may be considered as highly sensitive species to lantana and proportionality of these unique species increased on the nil lantana cover site with respect to differently covered lantana sites. Species that occurred ubiquitously, irrespective of lantana cover may be considered as species insensitive to lantana cover (Table 4).

Table 4. Importance value indices of herbaceous species under nil, low, medium and high lantana cover and species information

\begin{tabular}{|c|c|c|c|c|c|c|c|c|}
\hline \multirow[b]{2}{*}{ Species } & \multirow[b]{2}{*}{ Family } & \multirow[b]{2}{*}{$\begin{array}{l}\text { Life } \\
\text { form }\end{array}$} & \multirow[b]{2}{*}{$\mathbf{A} / \mathbf{P}$} & \multirow[b]{2}{*}{ N/NN } & \multicolumn{4}{|c|}{ Lantana cover $(\%)$} \\
\hline & & & & & $\begin{array}{l}\text { Nil } \\
(0 \%)\end{array}$ & $\begin{array}{c}\text { LOW } \\
(0-30 \%)\end{array}$ & $\begin{array}{c}\text { MEDIUM } \\
(31-60 \%)\end{array}$ & $\underset{(61-100 \%)}{\text { HIGH }}$ \\
\hline Alysicarpus vaginalis (L.) DC & Fabaceae & Th & A & $\mathrm{N}$ & 8.76 & 0.00 & 0.00 & 0.00 \\
\hline Andrographis echioides Nees. & Acanthaceae & Th & A & $\mathrm{N}$ & 5.29 & 0.00 & 0.00 & 0.00 \\
\hline Anagallis arvensis $\mathrm{L}$. & Primulaceae & Th & A & $\mathrm{NN}$ & 0.00 & 0.00 & 1.17 & 0.00 \\
\hline Barleria cristata $\mathrm{L}$. & Acanthaceae & $\mathrm{H}$ & $\mathrm{P}$ & $\mathrm{N}$ & 0.00 & 0.00 & 0.65 & 0.00 \\
\hline Begonia picta $\mathrm{Sm}$. & Begnoniaceae & $\mathrm{H}$ & A & $\mathrm{N}$ & 3.96 & 0.00 & 0.00 & 0.00 \\
\hline Cassia tora $\mathrm{L}$. & Caesalpiniacae & $\mathrm{Th}$ & A & $\mathrm{NN}$ & 3.02 & 1.01 & 2.79 & 3.75 \\
\hline Ceropegia bulbosa $\mathrm{L}$. & Asclepediaceae & G & $\mathrm{P}$ & $\mathrm{N}$ & 2.83 & 0.00 & 0.00 & 0.00 \\
\hline Chloris dolichostachya Lag. & Poaceae & $\mathrm{H}$ & $\mathrm{P}$ & $\mathrm{N}$ & 0.00 & 0.53 & 0.00 & 0.74 \\
\hline
\end{tabular}


Table 4. cont.

\begin{tabular}{|c|c|c|c|c|c|c|c|c|}
\hline \multirow[b]{2}{*}{ Species } & \multirow[b]{2}{*}{ Family } & \multirow{2}{*}{$\begin{array}{l}\text { Life } \\
\text { form }\end{array}$} & \multirow[b]{2}{*}{$\mathbf{A} / \mathbf{P}$} & \multirow[b]{2}{*}{$\mathbf{N} / \mathbf{N N}$} & \multicolumn{4}{|c|}{ Lantana cover $(\%)$} \\
\hline & & & & & $\begin{array}{c}\text { Nil } \\
(0 \%)\end{array}$ & \begin{tabular}{|c|c|} 
LOW \\
$(0-30 \%)$
\end{tabular} & \begin{tabular}{|c} 
MEDIUM \\
$(31-60 \%)$ \\
\end{tabular} & $\begin{array}{l}\text { I HIGH } \\
(61-100 \%)\end{array}$ \\
\hline $\begin{array}{l}\text { Chryanthellum americanum (L.) } \\
\text { Vatke }\end{array}$ & Poaceae & Th & A & NN & 0.00 & 0.00 & 2.30 & 1.84 \\
\hline Commelina benghalensis $\mathrm{L}$. & Commelinaceae & $\mathrm{H}$ & $\mathrm{P}$ & $\mathrm{N}$ & 0.00 & 0.00 & 1.56 & 0.58 \\
\hline Corchorus trilocularis L. & Tiliaceae & Th & A & $\mathrm{N}$ & 4.71 & 10.15 & 14.60 & 15.96 \\
\hline $\begin{array}{l}\text { Coronopus didymus (Linn.) } \\
\text { Smith }\end{array}$ & Brassicaceae & Th & A & NN & 0.00 & 0.00 & 0.00 & 0.00 \\
\hline Cyanotis axillaris Schult. & Poaceae & Th & A & $\mathrm{N}$ & 5.87 & 0.00 & 0.00 & 0.00 \\
\hline Cynodon dactylon Pers. & Poaceae & $\mathrm{H}$ & $\mathrm{P}$ & $\mathrm{N}$ & 1.68 & 2.83 & 0.00 & 2.38 \\
\hline Cyperus cyperoides Kuntze. & Cyperaceae & $\mathrm{H}$ & $\mathrm{P}$ & NN & 2.77 & 1.90 & 6.02 & 0.00 \\
\hline Desmodium triflorum DC. & Fabaceae & $\mathrm{H}$ & A & $\mathrm{NN}$ & 4.10 & 11.10 & 5.32 & 11.48 \\
\hline Dichanthium annulatum Stapf & Poaceae & $\mathrm{H}$ & $\mathrm{P}$ & NN & 1.70 & 1.33 & 0.00 & 0.00 \\
\hline Digitaria ciliaris Koel. & Poaceae & Th & A & - & 5.66 & 7.52 & 11.03 & 3.59 \\
\hline Echinochloa colona Link. & Poaceae & The & A & $\mathrm{NN}$ & 0.00 & 0.00 & 0.00 & 0.72 \\
\hline Euphorbia hirta L. & Euphorbiaceae & Th & A & $\mathrm{N}$ & 0.00 & 0.00 & 0.79 & 0.00 \\
\hline Evolvulus alsinoides $\mathrm{L}$. & Convolvulaceae & $\mathrm{H}$ & $\mathrm{P}$ & NN & 5.94 & 12.72 & 3.45 & 5.54 \\
\hline Evolvulus nuтmularius L. & Convolvulaceae & $\mathrm{H}$ & $\mathrm{P}$ & $\mathrm{NN}$ & 3.14 & 8.63 & 6.13 & 8.32 \\
\hline Fimbristylis ferruginea $\mathrm{Vahl}$ & Cyperaceae & Th & A & NN & 1.37 & 0.91 & 0.00 & 2.96 \\
\hline Hyptis suaveolens (L.) Poit. & Lamiaceae & Th & A & NN & 2.02 & 0.67 & 2.62 & 0.00 \\
\hline Ichnocarpus frutescens (L.) R. Br. & Asclepediaceae & $\mathrm{Ch}$ & $\mathrm{P}$ & NN & 0.00 & 0.00 & 0.00 & 1.70 \\
\hline Justicia simplex Don. & Acanthaceae & Th & A & $\mathrm{N}$ & 3.17 & 0.00 & 0.00 & 0.00 \\
\hline Leucas aspera $\mathrm{Spr}$ & Lamiaceae & Th & A & $\mathrm{N}$ & 1.67 & 0.61 & 0.00 & 0.00 \\
\hline Lindernia ciliata Pennell. & Scrophulariaceae & Th & A & $\mathrm{N}$ & 6.59 & 0.00 & 0.00 & 0.00 \\
\hline Ludwigia perennis L. & Onagraceae & Th & A & NN & 0.00 & 1.47 & 1.95 & 5.02 \\
\hline Malvastrum tricuspidatum Gray. & Malvaceae & Th & A & NN & 2.27 & 0.76 & 2.32 & 0.92 \\
\hline Mukia maderaspatana Roem. & Cucurbitaceae & Th & A & $\mathrm{N}$ & 0.00 & 1.77 & 0.00 & 2.27 \\
\hline Oplismenus compositus Beaub. & Poaceae & $\mathrm{H}$ & $\mathrm{P}$ & NN & 2.80 & 7.04 & 20.82 & 6.84 \\
\hline Phyllanthus niruri L. & Euphorbiaceae & Th & A & NN & 3.34 & 2.82 & 0.00 & 7.81 \\
\hline Phyllanthus urinaria $\mathrm{L}$. & Euphorbiaceae & Th & A & $\mathrm{NN}$ & 2.81 & 1.32 & 0.00 & 1.19 \\
\hline Physalis minima $\mathrm{L}$. & Solanaceae & Th & A & NN & 0.00 & 0.00 & 1.56 & 0.00 \\
\hline Rungia pectinata Nees. & Acanthaceae & Th & A & $\mathrm{N}$ & 1.30 & 0.77 & 0.00 & 0.58 \\
\hline Setaria pumila R. \& S. & Poaceae & Th & A & NN & 0.00 & 0.00 & 1.37 & 0.00 \\
\hline Sida acuta Burm. F. & Malvaceae & Th & $\mathrm{P}$ & NN & 4.20 & 10.01 & 6.82 & 4.88 \\
\hline Sida cordifolia $\mathrm{L}$. & Malvaceae & $\mathrm{H}$ & $\mathrm{P}$ & $\mathrm{NN}$ & 5.79 & 5.00 & 4.39 & 1.16 \\
\hline Sida rhombifolia $\mathrm{L}$. & Malvaceae & $\mathrm{H}$ & $P$ & NN & 3.25 & 2.54 & 0.00 & 0.58 \\
\hline Sporobolus diander Beauv. & Poaceae & $\mathrm{H}$ & $\mathrm{P}$ & NN & 0.00 & 0.00 & 1.56 & 0.00 \\
\hline Tephrosia purpurea Pers. & Fabaceae & Th & $\mathrm{P}$ & NN & 0.00 & 1.03 & 0.00 & 0.00 \\
\hline Urena lobata $\mathrm{L}$. & Malvaceae & Th & $\mathrm{P}$ & $\mathrm{NN}$ & 0.00 & 5.58 & 0.76 & 9.18 \\
\hline
\end{tabular}

$\mathrm{A}=$ Annual, $\mathrm{P}=$ Perennial; $\mathrm{N}=$ Native, NN= Non-native (Jackson, 1895) and personal communication with Dr. U Dhar. Th = Therophyte, $\mathrm{H}=$ Hemicryptophyte, $\mathrm{G}=$ Geophytic, $\mathrm{Ch}=$ Chamaephytic 


\section{Discussion}

The presence of disturbance in the form of canopy openings increases resource availability and also modifies the microclimate, which is consistent with the disturbance patch invasion model (Gentle and Duggin, 1997). The model state that the removal of competitive biomass and disruption of inter specific competitive interactions creates patches of increased resources. In the present study area canopy openings, which resulted from local disturbance, create patches of greater light availability. Increase in light availability follows gradient of disturbance intensities. Light has long been recognized as an important plant resource (Maximov, 1929; Blankenship, 2002) that may interact with other plant resources to affect plant performance (Cole, 2003). The increase in light availability increases the overall performance of lantana particularly the growth rate (Duggin and Gentle, 1998). Moreover, Chandrashekar and Swamy (2002) also reported that light availability in relatively less canopy enhance the growth of individual lantana.

With increased growth rate, lantana proliferates luxuriantly, which, as demonstrated in this study results in changes in species composition and soil properties. The growth architecture of lantana is such that it prevents light infiltration to the ground. Resulting in marked heterogeneity in terms of irradiance beneath the lantana bush and affects species diversity beneath its canopy. Light availability on the forest floor has been recognized as a key factor that influences intrinsic traits of inhabiting species (Jones et al., 1994; Walters and Reich, 1996). The dense cover created by vertical stratification of lantana may reduce the intensity or duration of light under its canopy and thus decrease the herbaceous cover. This could be due to the creation of a photosynthetically inactive light regime at ground level (Fetcher et al., 1983; Turton and Duff, 1992). Below certain thresholds, however, light limitation alone can prevent herbaceous species survival regardless of other resource levels (Tilman, 1982). It is likely that herbs are influenced by the amount of light that reaches the forest floor, and this may be probably one of the mechanisms responsible for the decline of herbaceous vegetation. Sharma and Raghubanshi $(2006,2007)$ advocated that the growth architecture pattern of lantana is such that it prevents the light penetration to the forest floor, leading to the decline of tree seedlings and possibly the herb flora.

Species like Anagallis arvensis L, Barleria cristata L., Dichanthium annulatum Stapf, Physalis minima L., Setaria pumila R. \& S., Sporobolus diander Beauv. with limited distribution as the lantana cover increases can be more susceptible to loss from physical damage or altered habitat conditions (Meier et al., 1995). Species like Dichanthium annulatum Stapf, Evolvulus alsinoides L., Leucas aspera Spr, Malvastrum tricuspidatum Gray., Rungia pectinata Nees., Sida acuta Burm. F., Sida cordifolia L., Sida rhombifolia L., Tephrosia purpurea Pers. which decrease and ultimately get locally extinct with increasing level of lantana cover may not recover quickly because of the altered physical environmental conditions (Roberts and Gilliam, 1995). Species which are unique (Alysicarpus vaginalis (L.) DC, Andrographis echioides Nees., Begonia picta Sm., Ceropegia bulbosa L., Cyanotis axillaris Schult., Justicia simplex Don. and Lindernia ciliata Pennell.) to no lantana cover sites are native species and are highly sensitive to environmental perturbations, are of great concern.

Lantana also possesses the capability to trap wind blown litter. This trapping of litter is also dependent on lantana cover, as denser the lantana cover, greater the trapping potential. So, more organic matter accumulates/builds up with increasing lantana cover. 
Deposition of litter due to wind also affects the herbaceous vegetation (Everham and Brokaw, 1996).

Accumulation of litter beneath the lantana canopies builds up soil organic matter. Accumulation of soil $\mathrm{N}$ closely follows that of soil organic matter because, on average $99 \%$ of the $\mathrm{N}$ in terrestrial ecosystem is organically bound (Rosswall, 1976). Raghubanshi (1992) reported strong positive relation between total $\mathrm{N}$ content and organic $\mathrm{C}$ content of soil in the dry deciduous forest ecosystem. According to Rawat et al., (1994) superiority in $\mathrm{N}$ extraction from the soil along with an efficient retranslocation of $\mathrm{N}$ from the senescing leaves enables lantana to perform better as an invasive species. Several studies have shown that soil nutrient levels play an important role in determining community invasibility (Shea and Chesson, 2002; Reinhart and Callaway, 2006). This self perpetuating changed microhabitat could probably provide lantana with increased resource leading to its successful proliferation.

In conclusion the presence of L. camara in the dry deciduous forest of India alters the spatial pattern of herbaceous layer vegetation and also changes the microhabitat conditions which could probably help towards its successful proliferation.

Acknowledgements. We thank Prof. J S Singh, Department of Botany, Banaras Hindu University, Varanasi for his thoughtful comments and suggestion in preparation of the manuscript. Funding support from Department of Science and Technology, and from Council of Scientific and Industrial Research, New Delhi, in form of a JRF to GPS is gratefully acknowledged.

\section{REFERENCES}

[1] Batianoff, G.N., Butler, D.W. (2003): Impact assessment and analysis of sixty-six priority invasive weeds in southeast Queensland. - Pl. Prot. Quart. 18: 11-17.

[2] Blankenship, R.E. (2002): Molecular Mechanisms of Photosynthesis. - Blackwell Science, Oxford.

[3] Carey, J.R., Moyle, P.B., Rejmànek, M., Vermeij, G. (1996): Preface. - Biol. Conserv. $78: 1$.

[4] Chandrasekaran, S., Swamy, P.S. (2002): Biomass, litterfall and above ground net primary productivity of herbaceous communities in varied ecosystems at kodayar in the western ghats of Tamilnadu. - Agric. Ecosyst. Environ. 88: 61-71.

[5] Cole, P.G. (2003): Environmental Constraints on the Distribution of the Non-native Invasive Grass, Microstegium vimineum. - PhD Thesis, Department of Ecology and Evolutionary Biology, University of Tennessee, Knoxville, TN

[6] Drake, J.A., di Castri, F., Grooves, R.H., Druger, F.J., Mooney, H.A., Rejmanek, M., Williamson, M. (eds.) (1989): Biological Invasion: a Global Perspective. - SCOPE 37. John Wiley \& Sons Ltd., Chichester, UK, pp 525

[7] Duggin, J.A., Gentle, C.B. (1998): Experimental evidence on the importance of disturbance intensity for invasion of Lantana camara L. in dry rainforest-open forest ecotones in north-eastern NSW, Australia. - For. Ecol. Manag. 109: 279-292.

[8] Everham, E.M., Brokaw, N.V.L. (1996): Forest damage and recovery from catastrophic wind. - Bot. Rev. 62: 114-185.

[9] Fetcher, N., Strain, B.R., Oberbauer, S.F. (1983): Effects of light regime on the growth, leaf morphology, and water relations of seedlings of two species of tropical trees. Oecologia 58: 314-319. 
[10] Gentle, C.B., Duggin, J.A. (1997): Lantana camara L. invasions in dry rainforest open forest ecotones: The role of disturbances associated with fire and cattle grazing. - Aust. J. Ecol. 22: 298-306.

[11] GISP (2003): The IAS problem. The Global Invasive Species Programme - Retrieved from http://www.gisp.org/about/IAS.asp on 28 December 2004

[12] Islam, K.R., Ahmed, M.R., Bhuiyan, M.K., Badruddin, A. (2001): Deforestation effects on vegetative regeneration and soil quality in tropical semi-evergreen degraded and protected forests of Bangladesh. - Land Degrad. Dev. 12: 45-56.

[13] Jackson, B.D. (1895): Index Kewensis. - Clarendon Press, Oxford, UK.

[14] Jackson, M.L. (1958). Soil chemical analysis. - Prentice-Hall, Englewood Cliffs, NewYork.

[15] Jha, C.S., Singh, J.S. (1990): Composition and dynamics of dry tropical forests in relation to soil texture. - J. Veg. Sci. 1: 609-614.

[16] Jones, R.H., Sharitz, R.R., Dixon, P.M., Segal, D.S., Schneider, R.L. (1994): Woody plant regeneration in four floodplain forests. - Ecol. Mono. 64: 345-367.

[17] Maximov, N.A. (1929): The Plant in Relation to Water. - George Allen \& Unwin, London.

[18] McAleece, N. (1997): Biodiversity Pro. - Available from http://www.sams.ac.uk/dml/projects/benthic/bdpro on 25 December 2004.

[19] Meier, A.J., Bratton, S.P., Duffy, D.C. (1995): Possible ecological mechanisms for loss of vernal-herb diversity in logged eastern deciduous forests. - Ecol. Appl. 5: 935-946.

[20] MoEF (1999): National policy and macro-level action strategy on biodiversity. - Ministry of Environment and Forest, Government of India, New Delhi, India.

[21] Mueller-Dumbois, D., Ellenberg, H. (1974): Aims and methods of vegetation ecology. John Wiley \& Sons, New York.

[22] Phillips, E.A. (1959): Methods of Vegetation Study. - Henry Holt and Company, Inc. $107 \mathrm{pp}$

[23] Pimm, S.L. (1986): Community structure and stability. - In: Soule M (ed) Conservation Biology: The Science of Scarcity and Diversity. Sinauer, Sunderland, Massachusetts, USA. pp 309-329.

[24] Ragubanshi, A.S. (1992): Effect of topography on selected soil properties and nitrogen mineralization in a dry tropical forest. - Soil Biol. Biochem. 24(2): 145-150.

[25] Rawat, Y.S., Bhatt, Y.D., Pande, P., Singh, S.P. (1994): Production and nutrient cycling in Arundinaria falcata and Lantana camara: The two converted ecosystems in central Himalaya. - Trop. Ecol. 35(1): 53-67.

[26] Reinhart, K.O., Callaway, R.M. (2006): Soil biota and invasive plants. - New Phytol. 170: 445-457.

[27] Roberts, M.R., Gilliam, F.S. (1995): Patterns and mechanism of plant diversity in forested in forested ecosystems: implications for forest management. - Ecol. Appl. 5: 969-977.

[28] Rosswall, T. (1976): The internal nitrogen cycle between microorganisms, vegetation and soil. - In: Svensson B H, Soderlund R (eds): Nitrogen, Phosphorous and Sulfur-Global Cycle, Ecol. Bull., Stockholm. pp 157-167

[29] Sharma, G.P, Raghubanshi, A.S. (2006): Tree population structure, regeneration and expected future composition at different levels of Lantana camara L. invasion in the Vindhyan tropical dry deciduous forest of India. - Lyonia. 11(1): 25-37.

[30] Sharma, G.P., Singh, J.S., Raghubanshi, A.S. (2005a): Plant invasions: emerging trends and future implications. - Curr. Sci. 88: 726-734.

[31] Sharma, G.P., Raghubanshi, A.S., Singh, J.S. (2005b): Lantana invasion: an overview. Weed Bio. Mgmt. 5: 157-167. 
[32] Sharma, G.P., Raghubanshi, A.S. (2007): Effect of Lantana camara L. cover on plant species depletion in the Vindhyan tropical dry deciduous forest of India. - Applied Ecology and Environmental Research 5 (1): 109-121

[33] Sharma, O.P. (1988):. How to combat lantana (Lantana camara L.) menace? - A current perspective. - J. Sci. Indust. Res. 47: 611-616.

[34] Shea, K., Chesson, P. (2002): Community ecology theory as a framework for biological invasions. - Trends Ecol. Evol. 17(4): 170-176.

[35] Singh, J.S., Singh, V.K. 1992. Phenology of seasonally dry tropical forest. - Curr. Sci. 63: 684-688.

[36] Singh, V.P., Singh, J.S. (1989): Man and forest: a case study from the dry tropics of India. - Environ. Conser. 16: 129-136.

[37] Singh, A. K., Raghubanshi, A. S., Singh, J. S. (2002): Medical ethnobotany of the tribals of Sonaghati of Sonebhadra district, Uttar pradesh, India. $J$. Ethnopharmacology 81: 31-41.

[38] SPSS (1997): SPSS Base7.5 Application Guide. - SPSS, Chicago, 389pp

[39] Tilman, D. (1982): Resource Competition and Community Structure. - Princeton University Press, NJ.

[40] Tripathi A (2003): Community Diversity Assesment in Vindhyans. - Ph. D. Thesis, Department of Botany, Banaras Hindu University, Varanasi-221005, India.

[41] Troup RS (1921): The Silviculture of Indian Trees, Vol. I and II. - Oxford: Clarendon Press.

[42] Turton SM, Duff GA (1992): Light environments and floristic composition across an open forest-rainforest boundary in northeastern Queensland. - Aust. J. Ecology 17: 415423

[43] Walters MB, Reich PB (1996): Are shade tolerance survival and growth linked? Low light and nitrogen effects on hardwood seedlings. - Ecology 77: 841-853. 